\title{
COVID-19: From Epidemic to Pandemic
}

\author{
James J James, MD, Dr PH, MHA
}

$\mathrm{J}$ ust after I finished my last editorial ${ }^{1}$, WHO declared COVID-19 to be a pandemic, ushering in a new wave of heightened anxiety, public panic, and global socio-economic damage. The extreme measures implemented in order to combat this public health emergency may well have put us on the cusp of a worldwide depression. Unfortunately, we have yet to meaningfully consider whether the disease or our response to it is having the more negative impact on the public's health. Such an assessment is critical, and it needs to be undertaken yesterday. Sadly, it was not. Although we are now in a position of catch-up, it remains our obligation as healthcare professionals to inform the way forward. We must support mitigating strategies based on what we know best-sound medical and public health strategies supported by data and experience.

Rather than reiterate and restate what has already been too frequently reported and overly dissected, I will use this editorial to discuss six important aspects of the COVID-19 pandemic and our responses to it that, I feel, warrant immediate consideration. Relevant observations along with suggestions for going forward will be presented, focusing on the U.S. experience where appropriate. As we proceed, one caveat: we all have to recognize that although we have significantly increased our knowledge of the virology, the epidemiology and the clinical aspects of this disease, we still have even more to learn. Too many questions cannot be answered because we do not yet have sufficient knowledge and understanding to do so.

\section{PREDICTIVE EPIDEMIOLOGICAL MODELS}

Over the past several weeks, a number of models predicting the number of COVID-19 cases and associated deaths have been published by government agencies and academic institutions. Many have predicted upwards of a hundred million cases resulting in over one million U.S. deaths in worst-case scenarios. These numbers have been repeatedly broadcast to the public, instilling fear and inciting panic. The predictions have been reported and received with a level of assumed veracity that is little deserved. Our limited understanding of the transmission characteristics of COVID-19, our lack of knowledge regarding subclinical cases of infection, the unknown relative number of susceptible versus non-susceptible individuals, and a

lack of understanding of agent infectivity, make it impossible to calculate statistical estimates with any degree of accuracy. To further extrapolate inaccurate estimates into the future without consideration of possible public health and medical interventions is an exercise in futility. To fashion policies around such predictive estimates invites folly. We seemingly refuse to learn from history; the modelers in 2014 predicted well over 1 million Ebola cases while in the final analysis approximately 30 thousand were reported. Rather than relying on these pseudo-scientific constructs, we should look to several real life COVID-19 epidemic models from China, Italy, South Korea, and elsewhere with data and outcome measures to guide us. These critically analyzed models of actual outbreaks could potentially provide us with better insight into whole nation impacts than predictive COVID-19 modeling, limited as the latter is by our current level of knowledge.

\section{TRANSMISSION}

Empirically, it is logical and well accepted that, as a Corona virus, the primary mode of transmission for COVID-19 is via respiratory droplets. Fomites may also serve as an efficient mechanism, but significant questions regarding transmission remain unanswered. For the respiratory route, is there a minimal infectious dose for a given susceptible to become positive? Does that minimum dose vary by age in a dose-response sense? Likewise, does the degree of viral shedding correlate with the severity of the clinical expression of disease? As to fomites serving as a source of transmission, we do know that the virus can survive in the environment for several hours, but what is its actual viability and transmissibility in tissue culture and in vivo? What concentration remains viable under what environmental conditions? The answers to these questions are critical to making informed decisions about the best community tactics for mitigating the spread of COVID-19. Most specifically, knowing the extent of broad community transmission versus the role of highly efficient or super transmitters could greatly help define risk profiles, which would facilitate tailoring a more efficient and effective response.

\section{CASE DEFINITION}

If there is a single logical error that we consistently make in the biomedical sciences, it is dichotomizing 
continuous variables. With COVID-19, we divide the population into cases and non-cases without the availability of a more discrete, validated classification measure. We further compound the problem by using a case definition based on a positive test that, in essence, gives equal weighting regardless of clinical severity. If we are going to craft valid public health interventions and supporting policies, we need to, at a minimum, report positives as subclinical, clinical non-hospital, hospitalized and hospitalized-severe/critical. This graded scale provides a much more informative depiction of what is happening, and when incorporated into risk analysis can allow for more targeted individual and community interventions.

\section{TESTING}

The significant issues with the production, deployment, and availability of COVID-19 testing have been covered in great detail elsewhere, and the importance of improving the program is generally agreed upon. The critical need to increase testing in order to expand population surveys has been less emphasized, but it is equally important. Population surveys would provide the crucial information necessary to quantify the overall community prevalence of COVID-19. This information would make it possible to more accurately estimate the level of herd immunity, the overall percentage of non-susceptibles in a population needed to prevent further transmission and result in the epidemic dying out. The herd immunity level is directly related to the Ro, or basic reproductive number, which for COVID-19 has been estimated to be 2-3.The corresponding levels of herd immunity required would be $50 \%$ and $66 \%$ and this would greatly enhance our ability to target future immunization programs. Knowledge of these values would also allow for more flexible intervention policies. Population surveys would also help determine the role of super-spreading in transmission, which could further shape policy and lessen the need for blanket restrictions. It should also be noted that testing to identify subclinical cases of COVID-19 infection, not initially identified, will require antibody testing, which has now become available, and will greatly enhance our understanding of this pandemic and allow for more targeted and data-based interventions.

\section{RISK}

As the COVID-19 pandemic accelerates, increasingly draconian measures are being taken to enforce social distancing in order to decrease exposure and flatten the epidemiological curve, including the looming threat of a nationwide lockdown in the U.S. We need to step back and reassess our strategies. We are currently taking a dichotomized, one size fits all approach, without first trying to establish some sort of risk gradient that could be used to better target individual and community measures at the national, state and local levels. Blanket strategies fail to take into account significantly different risks for diverse populations and communities that vary across regions by geography and population density. Our experience with COVID-19 to date clearly allows for the identification of distinct risk categories, notably by age, but, in spite of this, we are applying broad containment and mitigation strategies based on worst-case scenarios across the nation and individual states irrespective of clear and distinct differentials. Disaster risk management has evolved significantly over recent years. We need to consult and utilize this well-trained cadre of professionals available to us. These experts, in collaboration with medical and public health professionals, could help in devising state-level programs based on the sound principles of risk analysis, risk weighting, scaling, and measured interventions. They could define and quantify low, moderate, and severe risk categories, each matched to recommended interventions that would provide an alternative, potentially far less harmful approach to the shotgun methodology we are currently employing. We need to be especially cautious as to the effectiveness of national or regional lockdowns for three very important reasons: 1) COVID-19 is already widespread across the U.S.; you cannot contain what has already escaped; 2) a complete lockdown is impossible to achieve as certain individual and vital community level activities cannot be curtailed; and 3) the lockdown of Italy has had limited demonstrable effect in halting the terrible progression of COVID-19 in that country in contrast to South Korea's approach, which has had admirable success in controlling the pandemic without imposing such an extreme measure.

\section{SOCIAL DETERMINANTS OF HEALTH (SDH)}

For the past 15 years, there has been a tremendous interest in and focus on SDH at both the global and national levels. The concept is driven by health equity. There is a strong, validated consensus that $\mathrm{SDH}$ are directly and strongly linked to population health status in terms of morbidity and mortality across a broad spectrum of infectious and chronic diseases, with income, education, and employment/unemployment ranking consistently as the most significant $\mathrm{SDH}$. The current COVID-19 mitigation strategies being imposed have already and will almost certainly continue to negatively impact each of these determinants. It is important to consider the effects this will have in years to come on population and individual health resulting from these impacts. This is not to minimize the need for COVID-19 interventions to protect health and lives; it is simply to underscore the need to consider that there will be a significant negative impact on these $\mathrm{SDH}$, the cost of which, history consistently demonstrates, will exceed the benefits derived. The operation will have been successful, but the patient will have died.

In order to reconcile the above, we can again turn to disaster risk management and something we are all familiar with, the disaster cycle and two of its components: response and recovery. Response begins with impact and recovery begins with response. As to COVID-19, we have certainly had impact, and we are in active response; but we have not yet addressed, nor begun, our recovery. If we continue our extreme 
interventions without mitigation, we will cause profound socio-economic damage that will set health equity back decades and spawn increased rates of drug and alcohol dependence, crime, poverty, mental health illness, and a host of associated chronic medical conditions. The documented boom in alcohol sales across the country is but a harbinger of the negative behaviors we will experience.

We need to take action now and begin the necessary recovery process. This does not mean eschewing our COVID-19 interventions. They are vitally important to protect our people and our health system. It does mean using the many risk management tools we have at our disposal to find a balance. This balance can be achieved, but we must have a sustained commitment to recovery-recovery of our patients and recovery of our socio-economic support system. We are strong enough to do both; we have too much at risk to do otherwise.

\section{REFERENCE}

1. James JJ. COVID-19: A Tale of Two Epidemics. Disaster Med Public Health Prep. 2020;14(6):e1-e2. doi: 10.1017/dmp.2020.58 